\title{
LOCAL ANISOTROPY ESTIMATION FROM qP-WAVE VSP DATA: ANALYSIS OF 3D SURVEY DESIGN
}

\author{
Bruno dos Santos Silva ${ }^{1}$ and Ellen de Nazaré Souza Gomes ${ }^{1,2}$
}

\begin{abstract}
In the world, several unconventional hydrocarbon reservoirs have been found. This type of reservoir generally has anisotropic properties. The estimation of the anisotropy of the medium can give useful information about the reservoir, for example, one can obtain the information on the direction of fractures that are related to the preferential flow. This information is important in deciding which direction to drill the well. Measurements of slowness and polarization of $q P$-wave obtained from VSP (vertical seismic profile) experiments allow estimating the anisotropy in the vicinity of a geophone inside the borehole. Using the perturbation theory, a weakly anisotropic medium can be modeled by first-order perturbation around a reference isotropic medium. This approach allows to obtain a linear approximation which expresses the slowness and polarization in terms of WA (weak anisotropy) parameters. These parameters characterize the deviations of the anisotropic medium from a reference isotropic medium. In presented inversion scheme, we use the three components of the polarization, since we consider 3C (three-components) geophones, and only one of the slowness components, the one along the borehole direction, in which the receiver array is located. In this work, the inversion scheme using VSP data of slowness and polarization from direct $q P$-wave for the estimation of the parameters of weak anisotropy is analyzed considering the orientation of the horizontal borehole. Three different configurations for the sources are analyzed. The results are compared with results from vertical borehole. It has been found that only a group of components of the tensor of the WA parameters is well estimated and this group depend on the orientation of the borehole. On the other hand, the phase velocity determined from the WA parameter tensor is always well estimated in a $30^{\circ}$ cone around the borehole, regardless of the borehole orientation.
\end{abstract}

Keywords: local anisotropy, VSP multiazimuthal, linear inversion, survey design.

RESUMO. Muitos reservatórios de hidrocarbonetos não convencionais têm sido encontrados. Esse tipo de reservatório geralmente tem propriedades anisotrópicas. A estimativa da anisotropia do meio pode fornecer informações úteis sobre o reservatório como, por exemplo, a direção das fraturas, as quais estão relacionadas à direção de fluxo preferencial. Logo, esta informação é importante para decidir a direção de perfuração de um poço. Medidas de vagarosidade e polarização de ondas $q P$ obtidas em levantamentos de VSP (vertical seismic profile) permitem estimar a anisotropia na vizinhança de um geofone dentro do poço. Usando a teoria da perturbação, um meio fracamente anisotrópico pode ser modelado como uma perturbação de primeira ordem em relação a um meio isotrópico de referência. Esta abordagem possibilita a obtenção de uma aproximação linear que expressa a vagarosidade e polarização em termos de parâmetros WA (fraca anisotropia). Esses parâmetros caracterizam 0 desvio do meio anisotrópico em relação a um meio isotrópico de referência. No esquema de inversão são usadas as três componentes do vetor de polarização, pois considera-se geofones $3 \mathrm{C}$ (três componentes), e apenas uma componente do vetor de vagarosidade, a componente ao longo da direção de orientação do poço, no qual estão localizados os receptores. Neste trabalho é analisado um esquema de inversão para a estimativa de parâmetros anisotrópicos, são usados dados de vagarosidade e polarização de ondas $q P$ diretas em experimentos de VSP considerando a orientação do poço horizontal. Três diferentes configurações para as fontes são estudadas. Os resultados foram comparados com os resultados obtidos considerando o poço vertical. Verificou-se que apenas um grupo de componentes do tensor dos parâmetros elásticos WA é bem estimado. Este grupo depende da orientação do poço. Por outro lado, a velocidade de fase determinada a partir dos parâmetros WA é sempre bem estimada em um cone de $30^{\circ}$ entorno do poço, independentemente de sua orientação.

Palavras-chave: anisotropia local, VSP multiazimutal, inversão linear, desenho de experimento.

\footnotetext{
${ }^{1}$ Universidade Federal do Pará, Programa de Pós-Graduação em Geofísica, Rua Augusto Corrêa, 01, 66075-1100 Belém, PA, Brazil - E-mails: brunossgf@gmail.com, ellensgufpa@gmail.com

${ }^{2}$ Instituto Nacional de Ciência e Tecnologia - Criosfera, Brazil.
} 


\section{INTRODUCTION}

The anisotropy of a geological formation can be related to natural or induced factors. Among all the causes, we can highlight: natural fracturing induced by stress imbalance (tectonic effect) or artificial fractures (near to borehole) induced by hydraulic fracturing, compliant sediments (near to subsurface), layering (layers with width much smaller than the dominant wavelenght) and intrinsic (case of shales). The methodology presented here, can be useful to achieve the anisotropy degree introduced by any type of anisotropy. However, we are mainly interested in anisotropy induced by fractures (both natural and induced). Because by knowing the maximum degree of anisotropy, it possible to relate this magnitude with preferential direction of fractures and understand the three permeabilities in the fractured reservoir (Ehlig-Economides et al., 1990).

The understanding of the anisotropic reservoirs have play an important role on the Enhanced Oil Recovery (EOR) for this type of reservoir. For a 3D medium, the vertical seismic profiling (VSP) has been established as a technique for measuring in-situ anisotropy in hydrocarbon reservoir (Grechka et al., 2006; Tsvankin \& Grechka, 2011). From determination of anisotropic parameters that can be estimated from VSP data set, it is possible to enhance the resolution of seismic imaging, rock elastic property models and characterizing physical features of fractures or cracks (density, orientation, aspect ratio, etc).

Several techniques for estimation of anisotropy parameters from VSP data are found in the literature. In general terms, they can be categorized into two groups: methods that use only slownesses (Gaiser, 1990; Miller \& Spencer, 1994; Jílek et al., 2003) and methods that use polarization and slowness (Parscau, 1991; Horne \& Leaney, 2000; Dewangan \& Grechka, 2003; Grechka \& Mateeva, 2007). The application of each method depends primarily on the structural complexities in the overburden (Asgharzadeh et al., 2013).

Using first-order perturbation theory, Zheng \& Pšencík (2002) proposed a linearized model that relates perturbations of slowness and polarization of $q P$-wave to anisotropy parameters in weakly anisotropic media. This method does not depend of structural complexities in the overburden. Gomes et al. (2004) applied this approach to real VSP data collected in the Java Sea region.

The anisotropy estimation from VSP data are limited somehow by factors such as the survey geometry, noise level, data apertures or the wave types. Some recent studies have been concerned with analyzing and investigating the effect of these factors (Rusmanugroho \& McMechan, 2012; Barreto et al., 2013; Macambira et al., 2014; Ruzek \& Pšencík, 2016).

The design of VSP survey will define the illumination of the medium, hence, the information content in the data. Barreto et al. (2013), using the method of Zheng \& Pšencík (2002), investigated the design of multiazimuth walkaway surveys with vertical borehole and showed that at least five source profiles are required so that all anisotropy parameters related to $q P$-wave are independent in the inversion scheme. Recently, Ruzek \& Pšencík (2016), using a method that estimates the anisotropic parameters from $P$-wave traveltimes, showed that for this approach the use of sources distributed randomly on the surface improves substantially the parameter estimation.

In this work, we present a formulation for horizontal borehole which is an alternative form for vertical borehole formulation presented in Zheng \& Pšencík (2002). In this sense, this work is an extension of Barreto et al. (2013), the WA parameters are estimated from direct $q P$-wave data obtained in $3 \mathrm{D}$ VSP experiment where the receivers are distributed in a horizontal borehole and three distribution of sources on the surface are considered: along five radial profiles, randomly distributed (Ruzek \& Pšencík, 2016) and in spiral pattern (Blackburn et al., 2007). For comparison, the results obtained here, for the horizontal borehole, were compared with the results obtained for vertical borehole (Barreto et al., 2013).

As horizontal borehole are commonly drilled in unconventional reservoirs, this work can be useful in studies for this kind of reservoirs such as characterization of fracture and fluid content evaluation. For example: if we consider the anisotropic medium of the TI (Transverse Isotropy) type, through the anisotropy parameters we can determine the axis of symmetry of this medium and thus the direction of induced fracturing. The fracture direction is known to be the preferred direction of flow.

\section{METHODOLOGY}

The weak anisotropy medium is modeled by first-order perturbations around an isotropic reference medium. In the following, the Cartesian coordinate system $(x, y, z)$ is used for description of the model. The $z$-axis is chosen positive downwards and the positive $x$ - and $y$-axes are chosen so that the coordinate system is right-handed.

Let us consider a weakly anisotropic medium and take an isotropic medium as a reference one. The slowness vector $p_{i}$ of the $q P$-wave in a weakly anisotropic medium can be expressed as

$$
p_{i}=p_{i}^{0}+\Delta p_{i},
$$


or

$$
\begin{aligned}
p_{i} & =p_{i}^{0}+\Delta \xi \vec{i}+\Delta \zeta \vec{j}+\Delta \eta \vec{k} \\
& =(\xi+\Delta \xi) \vec{i}+(\zeta+\Delta \zeta) \vec{j}+(\eta+\Delta \eta) \vec{k}
\end{aligned}
$$

where $p_{i}^{0}$ is a slowness vector in the reference isotropic medium and $\Delta p_{i}$ is its perturbation. $\vec{i}, \vec{j}, \vec{k}$ are unit vectors along the axes $x, y$ and $z$, respectively. $\xi$, $\zeta$ and $\eta$ denote projection of the slowness vector $p_{i}^{0}$ onto $\vec{i}, \vec{j}, \vec{k}$, respectively. $\Delta \xi, \Delta \zeta$ and $\Delta \eta$ denote perturbations of $p_{i}^{0}$. The vector $p_{i}^{0}$ is given by

$$
p_{i}^{0}=\alpha^{-1} n_{i}
$$

and its components has the form

$$
\xi=\frac{n_{1}}{\alpha}, \quad \zeta=\frac{n_{2}}{\alpha}, \quad \eta=\frac{n_{3}}{\alpha},
$$

where $\alpha$ is the $P$-wave velocity and the vector $n_{i}=\left(n_{1}, n_{2}, n_{3}\right)$ represents the wave normal, both in the isotropic reference medium.

The wave normal and polarization vector of the $P$-wave are identical in an isotropic medium. Thus the polarization vector, $g_{i}$, of $q P$-wave in a weakly anisotropic can be written as:

$$
g_{i}=n_{i}+\Delta g,
$$

where $\Delta g$ is the deviation from the orientation of the polarization vector in a reference isotropic medium.

Let us introduce in the reference isotropic medium three mutually perpendicular unit vectors $e_{i}^{(1)}, e_{i}^{(2)}$ and $e_{i}^{(3)}$ so that the vector $e_{i}^{(3)}$ is identical with the wave normal of the $P$-wave $n_{i}$. A practical choice of vectors $e_{i}^{(1)}$ and $e_{i}^{(2)}$ expressed in terms of components of the vector $e_{i}^{(3)}$ is as follows (Pšencík \& Gajewski, 1998):

$$
\begin{aligned}
& e^{(1)}=D^{-1}\left(n_{1} n_{3}, n_{2} n_{3}, n_{3}^{2}-1\right), \\
& e^{(2)}=D^{-1}\left(-n_{2}, n_{1}, 0\right), \\
& e^{(3)}=n=\left(n_{1}, n_{2}, n_{3}\right),
\end{aligned}
$$

where

$$
D=\sqrt{n_{1}^{2}+n_{2}^{2}}, n_{1}^{2}+n_{2}^{2}+n_{3}^{2}=1 .
$$

Using the vectors $e_{i}^{(k)}$, Pšencík \& Gajewski (1998) defined the weak anisotropy matrix:

$$
B_{m n}=a_{i j k l} e_{i}^{(m)} e_{j}^{(3)} e_{l}^{(3)} e_{k}^{(n)}-c_{0}^{2} \delta_{m n},
$$

where $a_{i j k l}$ denotes the tensor of density-normalized elastic parameters, $c_{0}$ stands for the phase velocity of the reference isotropic medium, specified by the $P$ - and $S$-wave velocities $\alpha$ and $\beta$. For $m=n=3, c_{0}=\alpha$; for $m=n=1$ or 2 , $c_{0}=\beta$. The elements of the matrix $B_{m n}$ are linear function of weak anisotropy (WA) parameters. The WA parameters represent a generalization of Thomsen's parameters to anisotropic media of arbitrary symmetry and orientation (Pšencík \& Gajewski, 1998; Farra \& Pšencík, 2003). Propagation of $q P$-wave in weakly anisotropic medium is specified by 15 WA parameters, which are related to density-normalized elastic parameters in the Voigt notation $A_{\alpha \beta}$ in the following way:

$$
\begin{aligned}
\varepsilon_{x} & =\frac{A_{11}-\alpha^{2}}{2 \alpha^{2}}, & \varepsilon_{y} & =\frac{A_{22}-\alpha^{2}}{2 \alpha^{2}}, \\
\varepsilon_{z} & =\frac{A_{33}-\alpha^{2}}{2 \alpha^{2}}, & \delta_{x} & =\frac{A_{13}+2 A_{55}-\alpha^{2}}{\alpha^{2}}, \\
\delta_{y} & =\frac{A_{23}+2 A_{44}-\alpha^{2}}{\alpha^{2}}, & \delta_{z} & =\frac{A_{12}+2 A_{66}-\alpha^{2}}{\alpha^{2}}, \\
\chi_{x} & =\frac{A_{14}+2 A_{56}}{\alpha^{2}}, & \chi_{y} & =\frac{A_{25}+2 A_{46}}{\alpha^{2}}, \\
\chi_{z} & =\frac{A_{36}+2 A_{45}}{\alpha^{2}}, & \varepsilon_{15} & =\frac{A_{15}}{\alpha^{2}}, \\
\varepsilon_{16} & =\frac{A_{16}}{\alpha^{2}}, & \varepsilon_{24} & =\frac{A_{24}}{\alpha^{2}}, \\
\varepsilon_{26} & =\frac{A_{26}}{\alpha^{2}}, & \varepsilon_{34} & =\frac{A_{34}}{\alpha^{2}}, \\
\varepsilon_{35} & =\frac{A_{35}}{\alpha^{2}} . & &
\end{aligned}
$$

The slowness and polarization vectors of a $q P$-wave propagating in an arbitrary anisotropic medium are linearly related to the WA parameters of this medium through the equations (Zheng \& Pšencík, 2002):

$$
\begin{aligned}
B_{K 3}= & \left(\alpha^{2}-\beta^{2}\right)\left(g_{i} e_{i}^{(K)}-\alpha \Delta \xi e_{1}^{(K)}\right. \\
& \left.-\alpha \Delta \zeta e_{2}^{(K)}-\alpha \Delta \eta e_{3}^{(K)}\right), K=1,2
\end{aligned}
$$

$$
B_{33}=-2 \alpha^{4} \xi \Delta \xi-2 \alpha^{4} \zeta \Delta \zeta-2 \alpha^{4} \eta \Delta \eta
$$

The symbols $B_{13}, B_{23}$ and $B_{33}$ are elements of the weak anisotropy matrix $B_{m n}$, which depend on $15 q P$-wave WA 
parameters. These elements are written in the following form (Pšencík \& Gajewski, 1998):

$$
\begin{aligned}
B_{13}= & \alpha^{2} D^{-1}\left\{2 \varepsilon_{z} n_{3}^{5}+n_{3}^{4}\left(\varepsilon_{34} n_{2}+\varepsilon_{35} n_{1}\right)\right. \\
& +n_{3}^{3}\left(\delta_{x} n_{1}^{2}+\delta_{y} n_{2}^{2}+2 \chi_{z} n_{1} n_{2}-2 \varepsilon_{z}\right) \\
& +n_{3}^{2}\left[\left(4 \chi_{x}-3 \varepsilon_{34}\right) n_{1}^{2} n_{2}\right. \\
& +\left(4 \chi_{y}-3 \varepsilon_{35}\right) n_{1} n_{2}^{2}+\left(4 \varepsilon_{15}-3 \varepsilon_{35}\right) n_{1}^{3} \\
& \left.+\left(4 \varepsilon_{24}-3 \varepsilon_{34}\right) n_{2}^{3}\right]+n_{3}\left[\left(2 \delta_{z}-\delta_{x}-\delta_{y}\right) n_{1}^{2} n_{2}^{2}\right. \\
& +2\left(2 \varepsilon_{16}-\chi_{z}\right) n_{1}^{3} n_{2} \\
& \left.+\left(2 \varepsilon_{26}-\chi_{z}\right) n_{1} n_{2}^{3}+\left(2 \varepsilon_{x}-\delta_{x}\right) n_{1}^{4}+\left(2 \varepsilon_{y}-\delta_{y}\right) n_{2}^{4}\right] \\
& \left.-\chi_{x} n_{1}^{2} n_{2}-\chi_{y} n_{1} n_{2}^{2}-\varepsilon_{15} n_{1}^{3}-\varepsilon_{24} n_{2}^{3}\right\},
\end{aligned}
$$$$
B_{23}=\alpha^{2} D^{-1}\left\{n_{3}^{3}\left(\varepsilon_{34} n_{1}-\varepsilon_{35} n_{2}\right)\right.
$$$$
+n_{3}^{2}\left[\left(\delta_{x}+\delta_{y}\right) n_{1} n_{2}+\chi_{z} n_{1}^{2}-\chi_{z} n_{2}^{2}\right]
$$$$
+n_{3}^{2}\left[\left(2 \chi_{y}-3 \varepsilon_{15}\right) n_{1}^{2} n_{2}-\left(2 \chi_{x}-3 \varepsilon_{24}\right) n_{1} n_{2}^{2}\right.
$$$$
\left.+\chi_{x} n_{1}^{3}-\chi_{y} n_{2}^{3}\right]+\left(\delta_{z}-2 \varepsilon_{x}\right) n_{1}^{3} n_{2}
$$$$
+\left(2 \varepsilon_{y}-2 \delta_{z}\right) n_{1} n_{2}^{3}
$$$$
\left.+3\left(\varepsilon_{26}-\varepsilon_{16}\right) n_{1}^{2} n_{2}^{2}+\varepsilon_{16} n_{1}^{4}-\varepsilon_{26} n_{2}^{4}\right\},
$$$$
B_{33}=2 \alpha^{2}\left\{\varepsilon_{z} n_{3}^{4}+2 n_{3}^{3}\left(\varepsilon_{34} n_{2}+\varepsilon_{35} n_{1}\right)\right.
$$$$
+n_{3}^{2}\left(\delta_{x} n_{1}^{2}+\delta_{y} n_{2}^{2}+2 \chi_{z} n_{1} n_{2}\right)
$$$$
+2 n_{3}\left(\chi_{x} n_{1}^{2} n_{2}+\chi_{y} n_{1} n_{2}^{2}+\varepsilon_{15} n_{1}^{3}+\varepsilon_{24} n_{2}^{3}\right)+\varepsilon_{x} n_{1}^{4}
$$$$
+\delta_{z} n_{1}^{2} n_{2}^{2}+\varepsilon_{y} n_{2}^{4}
$$$$
\left.+2 \varepsilon_{16} n_{1}^{3} n_{2}+2 \varepsilon_{26} n_{1} n_{2}^{3}\right\} \text {. }
$$

Eqs. (10) and (11) state a linear relation between the WA parameters of the medium and the polarization and slowness vector. Thus this set of equations can be used for inversion of three-component $(3 \mathrm{C})$ data recorded in a receiver inside the borehole.

Let us consider a VSP experiment with receivers in a horizontal borehole along $x$-axis direction. In this case we have available only the $x$-component of the slowness vector, which is represented by $p_{1}=\xi+\Delta \xi$. If none of the perturbations $\Delta \eta$ and $\Delta \zeta$ is known we can obtain the equation for inversion by eliminating these perturbations from Eqs. (10) and (11). Eliminating firstly $\Delta \eta$ we obtain the set of equations:

$$
\begin{gathered}
\eta\left(\alpha^{2}-\beta^{2}\right)^{-1} B_{K 3}-\frac{1}{2} \alpha^{-3} B_{33} e_{3}^{(K)}= \\
\eta g_{i} e_{i}^{(K)}-\alpha \Delta \xi\left(\eta e_{1}^{(K)}-\xi e_{3}^{(K)}\right)-\alpha \Delta \zeta X^{(K)} \\
K=1,2
\end{gathered}
$$

where

$$
X^{(K)}=\eta e_{2}^{(K)}-\zeta e_{3}^{(K)},
$$

then we eliminate $\Delta \zeta$ from Eq. (15) and rearrange it in such way that we find the following form:

$$
\begin{gathered}
\left(\alpha^{2}-\beta^{2}\right)^{-1}\left(B_{13} e_{1}^{(1)}+B_{23} e_{1}^{(2)}\right)+\frac{1}{2} \alpha^{-1} B_{33} \xi= \\
g_{i}\left(e_{i}^{(1)} e_{1}^{(1)}+e_{i}^{(2)} e_{1}^{(2)}\right)-\alpha \Delta \xi .
\end{gathered}
$$

This proposed equation corresponds an alternative version of the approach of Zheng \& Pšencík (2002), but here we assume that the borehole is horizontal.

\section{Determination of the reference medium parameters}

Inversion Eq. (17) depend on isotropic reference parameters. The $P$-wave velocity, $\alpha$, of reference medium can be obtained from least-squares inversion of the following expression (Barreto et al., 2013):

$$
p_{i}=\alpha^{-1} g_{i} .
$$

Where $p_{i}$ and $g_{i}$ corresponds to slowness component observed ( $z$-component for vertical borehole and $x$-component for horizontal borehole) and polarization vector, respectively. The $S$-wave velocity, $\beta$, is determined by assuming that the reference medium is a Poisson solid, defined as:

$$
\beta=\frac{\alpha}{\sqrt{3}} .
$$

The wave normal vector $n_{i}$ is considered parallel to the polarization vector observed, so $n \| g$. This approximation is valid for weakly anisotropic medium.

\section{Inversion scheme}

WA parameters of the medium in the vicinity of borehole receivers can be estimated by inverting the appropriate equation for borehole orientation. For inversion procedure the suitable equation can be represented in matrix form:

$$
\mathbf{G} \mathbf{m}=\mathbf{d} .
$$

The symbol d represents a vector which is related to the observations, this vector is given by right side of used equation for each source-receiver pair and has dimension equal to the number of observations $\left(N_{\text {obs }}\right)$. Symbol $\mathbf{m}$ denotes the vector of model parameters, hence it consists of 15 WA parameters and has the form:

$$
\mathbf{m}=\left[\varepsilon_{x}, \varepsilon_{y}, \varepsilon_{z}, \delta_{x}, \delta_{y}, \delta_{z}, \chi_{x}, \chi_{y}, \chi_{z}, \varepsilon_{15}, \varepsilon_{16}, \varepsilon_{24}, \varepsilon_{26}, \varepsilon_{34}, \varepsilon_{35}\right]^{T}
$$


Finally, G represents a linear operator, called sensitivity matrix, which depends on the parameters of reference medium and the design of VSP experiment. The matrix $\mathbf{G}$ has dimension $N_{o b s} \times 15$ and its elements are obtained from left side of used equation.

Eq. (20) is solved by generalized inverse (Aster et al., 2019). The singular value decomposition (SVD) is used to compute the generalized inverse. Therefore, the solution can be written as

$$
\mathbf{m}^{\text {est }}=\mathbf{G}^{\dagger} \mathbf{d}
$$

or

$$
\mathbf{m}^{\mathrm{est}}=\mathbf{V S}^{-1} \mathbf{U}^{T} \mathbf{d},
$$

where $\mathbf{m}^{\text {est }}$ is the vector of estimated parameters, $\mathbf{G}^{\dagger}$ is the generalized inverse of $\mathbf{G}$. $\mathbf{U}$ and $\mathbf{V}$ are orthogonal matrices of eigenvectors that span the data space and model parameters space, respectively. $\mathbf{S}$ is the diagonal matrix whose diagonal elements are the singular values $\lambda_{1}, \lambda_{2}, \ldots, \lambda_{15}$.

\section{EXPERIMENTAL DESIGN}

For vertical borehole in multiazimuth walkaway VSP surveys, as shown by Barreto et al. (2013), at least five radial profiles are necessary so that all 15 WA parameters can be independently retrieved. Here we extend the analysis. For horizontal borehole three distribution of sources on the surface are considered: along five radial profiles, randomly distributed and in spiral pattern.

In the numerical experiments conducted in this work, the data are generated using the program package ANRAY (Gajewski \& Pšencik, 1990).

\section{Model and configuration of experiments}

The model consists of two layers confined in a box with dimensions $10 \mathrm{~km} \times 10 \mathrm{~km} \times 7 \mathrm{~km}$. The origin of cartesian coordinate system $(x, y, z)$ is situated in the center of the model. The interface between layers is located at a depth of $5 \mathrm{~km}$ and has a flat horizontal geometry (Fig. 1).

The first layer is heterogeneous anisotropic with anisotropy degree about $8 \%$. The elastic parameters at the top of the layer corresponds to VTI (transversely isotropy with a vertical symmetry axis) medium with symmetry axis rotated by $80^{\circ}$ around the $y$-axis and then $25^{\circ}$ around the $z$-axis. The elements of non-rotated matrix of the density-normalized elastic moduli in $(\mathrm{km} / \mathrm{s})^{2}$ of initial VTI medium is: $A_{11}=15.71, A_{13}=4.46$, $A_{33}=13.39, A_{44}=4.98$ and $A_{66}=5.33$ (Thomsen, 1986).

The elastic parameters at the bottom corresponds to VTI medium with symmetry axis rotated by $90^{\circ}$ around the $y$-axis. The elements of non-rotated matrix of the density-normalized elastic moduli in $(\mathrm{km} / \mathrm{s})^{2}$ of VTI medium is: $A_{11}=35.35, A_{13}=10.04$, $A_{33}=30.13, A_{44}=11.21$ and $A_{66}=11.99$ (Thomsen, 1986).

(a)

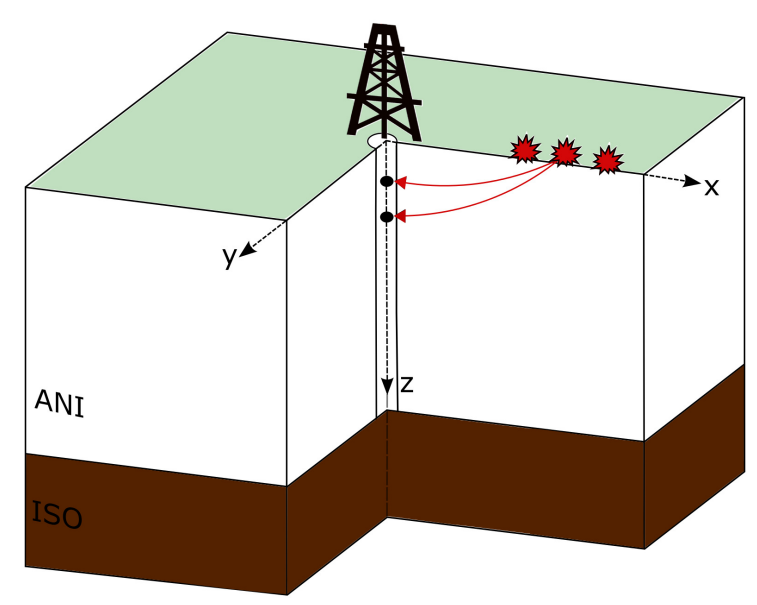

(b)

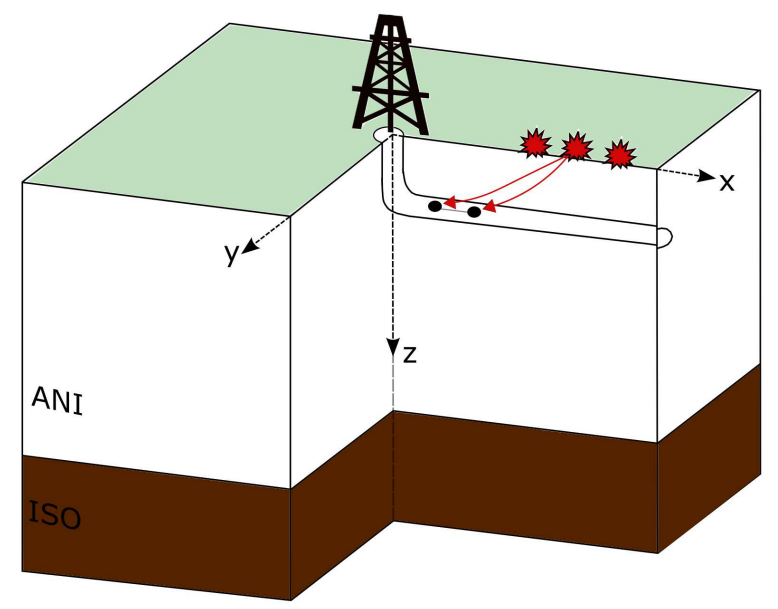

Figure 1-Schematic illustration of model with receiver in vertical and horizontal borehole. The first layer (ANI) is heterogeneous anisotropic and the second one (ISO) is homogeneous isotropic. (a) Vertical borehole with receivers in the $z$-axis direction. (b) Horizontal borehole with receivers parallel to the $x$-axis direction.

The distribution of elastic parameters (21 density-normalized elastic parameters) within this layer is given by linear interpolation between the values specified at the top $(z=0 \mathrm{~km})$ and at the bottom $(z=5 \mathrm{~km})$ surfaces. The second layer is a homogeneous isotropic medium, which is characterized by density-normalized $P$ - and $S$-wave velocities of $4.77 \mathrm{~km} / \mathrm{s}$ and $2.76 \mathrm{~km} / \mathrm{s}$, respectively.

At the comparison the estimates of the anisotropy obtained in a horizontal borehole will be presented together with the 
anisotropy estimates obtained in a vertical borehole. Information on the methodology and estimation of anisotropy for a vertical borehole applied here can be seen in Zheng \& Pšencík (2002); Gomes et al. (2004); Barreto et al. (2013) and Macambira et al. (2014).

In the vertical borehole configuration, the receivers array is in the $z$-axis direction (see Fig. 1a). In the horizontal borehole configuration, the receivers array is located at a depth of $0.5 \mathrm{~km}$ and parallel to $x$-axis direction (see Fig. 1b). The wellhead is situated in the origin of coordinate system.

The Figure 2 shows the three types of source distributions considered in the tests. In each experiment are used 180 sources. Sources close to the wellhead generate slowness vector that reach the well almost vertically, while sources farther from the wellhead generate slowness vector that reached the well almost horizontally. This may compromise the estimation of the anisotropy parameters, since in the inversion scheme the projection of the vector of slowness in the direction of the well is used.

Due to this, the distribution of the sources on the surface was made differently depending on the orientation of the borehole. For the horizontal borehole the sources were distributed in an area whose radius is the twice area where the sources were distributed for the vertical borehole.

In the first geometry (Fig. 2a), the sources are distributed along five radial profiles with angular steps of $72^{\circ}$, each profile contains 36 sources regularly spaced by $0.05 \mathrm{~km}$ for vertical borehole configuration or by $0.1 \mathrm{~km}$ for horizontal borehole. The second one consists of randomly distribution of sources (see Fig. $2 b)$. In the third distribution type is used a spiral pattern with dual sources array (see Fig. 2c).
The observed data comprises the three components of polarization and $x$-component of slowness (horizontal borehole) or $z$-component of slowness (vertical borehole) for each source-receiver pair. We use only direct $q P$-wave measurements.

\section{Sensitivity study}

A sensitivity analysis of WA parameters with respect to acquisition geometry is carried out by using the model resolution matrix. This tool determines whether model parameters can be independently predicted or calculated. The resolution matrix $\mathbf{R}$ can be calculated from singular value decomposition (SVD) of $\mathbf{G}$ in the following way (Menke, 2012):

$$
\mathbf{R}=\mathbf{V}_{r} \mathbf{V}_{r}^{T},
$$

where $\mathbf{V}_{r}$ is a submatrix of $\mathbf{V}$, that has dimension equal to the number of WA parameters. The matrix $\mathbf{V}_{r}$ is formed by the $r$ columns of $\mathbf{V}$ that are associated with the acceptable singular values, i.e., those greater than a specified cutoff value. This value is chosen by prescribing an acceptable condition number, defined as the ratio of the largest to smallest singular value. The analysis based on resolution matrix is conducted for receivers at depth of $0.5 \mathrm{~km}$.

Figure 3 shows the singular values computed for the three acquisition geometries in VSP experiment with horizontal borehole. It observed that for the three geometries none of singular values are smaller than 0.01 (cutoff value used). Since all the singular values are considered nonzero, the computation of the model resolution matrices yields identity matrices which means that each of 15 WA parameter can be uniquely determined for the three distributions. This result is similar to that found in the analysis for the vertical borehole. (a)

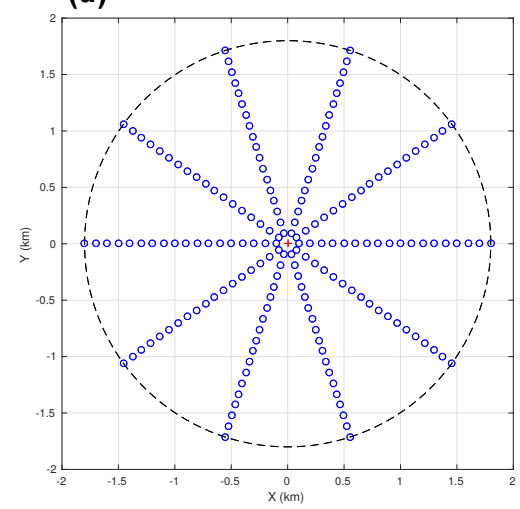

(b)

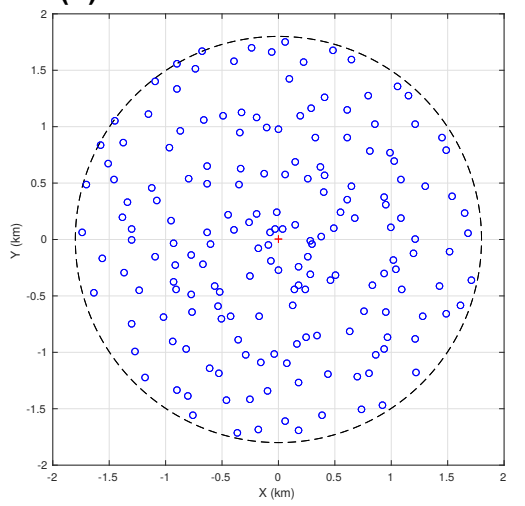

(c)

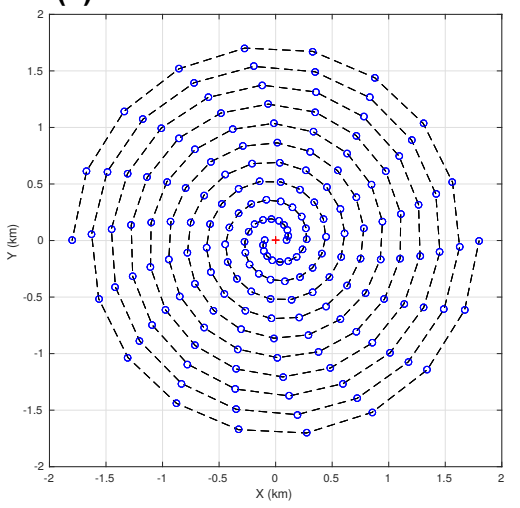

Figure 2 - Plan view of three types of source distribution considered. For each geometry 180 sources are used. The blue markers represent the source positions and the red marker the wellhead position. (a) sources along 5 profiles with angular steps of $72^{\circ}$. (b) sources randomly distributed. (c) sources in spiral pattern. 


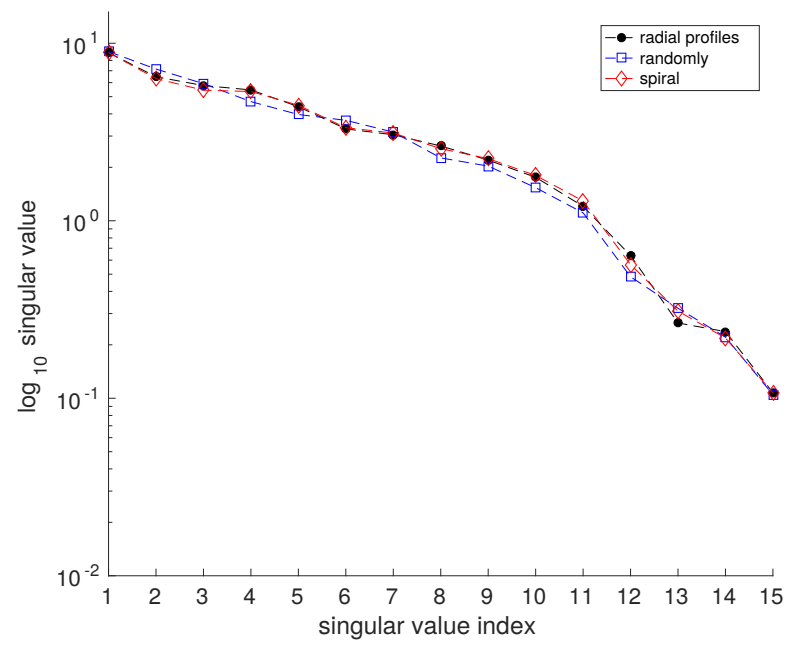

Figure 3 - Singular values for the three acquisition geometries (see Fig. 2) of VSP experiments with horizontal borehole. The black markers are values computed for the sources along five radial profiles. Blue markers are the results for sources randomly distributed. Red markers correspond the result for sources in spiral pattern. The similar result we have for vertical borehole.

The above analysis is a means to assessing which of sought parameters can be uniquely estimated using the acquisition geometries studied. Nonetheless, the resolution of estimates is affected by noise in data, hence it is necessary to investigate how errors in the data project errors in the estimated model. For this purpose, the model covariance matrix is computed in the inversion test.

\section{INVERSION TESTS}

The inversion scheme is apply for synthetic data contaminated with random Gaussian noise. The added noise has standard deviations of $1^{\circ}$ for polarization vector and $5 \%$ for slowness data ( $z$-component for vertical borehole configuration and $x$-component for horizontal configuration).

Tests are performed for data generated by the three types of source distribution (see Fig. 2) with each borehole configuration. For each data set, the inversion is carried out 500 times for different realizations of noise. The quality of WA parameters recovered from inversion is analyzed by computation of the first-order approximation of the phase velocity (Pšencík \& Gajewski, 1998) and comparing maps of phase velocity obtained with exact and inverted parameters. The first-order approximation of phase velocity is given by

$$
c\left(n_{i}, m_{j}\right)=\sqrt{\alpha^{2}+B_{33}},
$$

where $B_{33}$ is given by Eq. (14) and $\alpha$ stands for the $P$-wave velocity of the reference isotropic medium. This expression for phase velocity $c\left(n_{i}, m_{j}\right)$ depends on the wave normal vector $n_{i}$ and the model parameters vector $m_{j}$, see Eq. (21).

For presentation of inversion results, four types of stereographic projection maps are shown: (a) the phase velocity calculated from the "exact" WA parameters, using Eq. (25); (b) the phase velocity calculated from the expected WA parameters of 500 inversion trials; (c) the relative error expressed as a percent between (b) and (a); and (d) the percentage variation of the phase velocity. The latter map is obtained from the phase velocity calculated from estimates of the 500 inversions.

The stability of the WA parameter estimates is analyzed in two ways. In the first way, the analysis is done indirectly, through the variation of the phase velocities. In the second way, through the numerical computation of the model covariance matrix (Aster et al., 2019) by the following formula:

$$
\mathbf{C}_{i j}=\frac{1}{N} \sum_{k=1}^{N}\left(\mathbf{m}_{i}^{\text {est }(k)}-\overline{\mathbf{m}}_{i}\right)\left(\mathbf{m}_{j}^{\text {est }(k)}-\overline{\mathbf{m}}_{j}\right),
$$

where $\mathbf{C}_{i j}$ denotes the elements of covariance matrix, with $i, j=$ $1,2, \ldots, 15 . N$ stands for the number of different solutions $\mathbf{m}^{\text {est }}$ obtained from inversion trials. Finally, the symbol $\mathbf{m}$ corresponds to the vector of mean (expected) solution, which is obtained from the average of the $N=500$ solutions $\mathbf{m}^{\text {est }}$.

The diagonal entries of the model covariance matrix are the variances of estimated parameters and the off-diagonal elements describe the correlation between pairs of parameters. Since the noise in the data will cause errors in the model parameter estimates, the covariance values characterize the uncertainty in the recovered parameter.

For data set measured within the horizontal borehole, the inversion is performed using Eq. (17). In the tests it is considered the receiver located at $0.5 \mathrm{~km}$ depth, in the same direction of the positive $x$-axis and $0.4 \mathrm{~km}$ away from $z$-axis.

Before inversion procedure it is necessary to determine the velocities of the isotropic reference medium. The $P$-wave velocity are obtained from least-squares fitting of polarization and slowness components in the well direction, Eq. (18). The fitting procedure is carried out to data sets generated by the three source distributions. For each data set, the results corresponds to the mean of estimates for 500 realizations of random noise.

The velocities of the reference isotropic medium obtained from data sets generated by the three types of sources distribution are approximately $3.87 \mathrm{~km} / \mathrm{s}$ for $P$-wave and $2.23 \mathrm{~km} / \mathrm{s}$ for $S$-wave.

Figure 4 shows the stereographic maps of phase velocity computed using exact WA parameters (Fig. 4a), using 

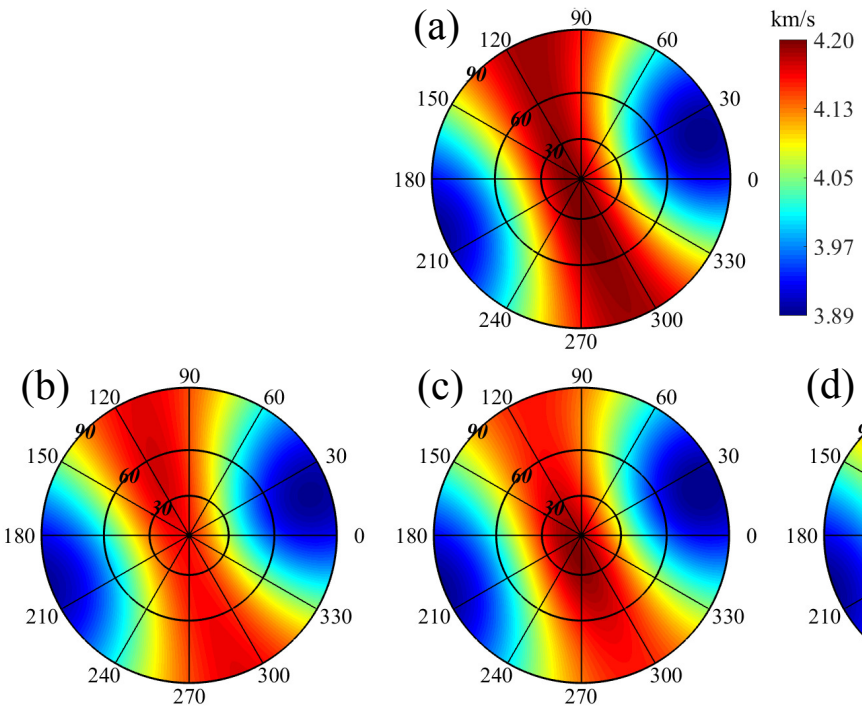

(d)
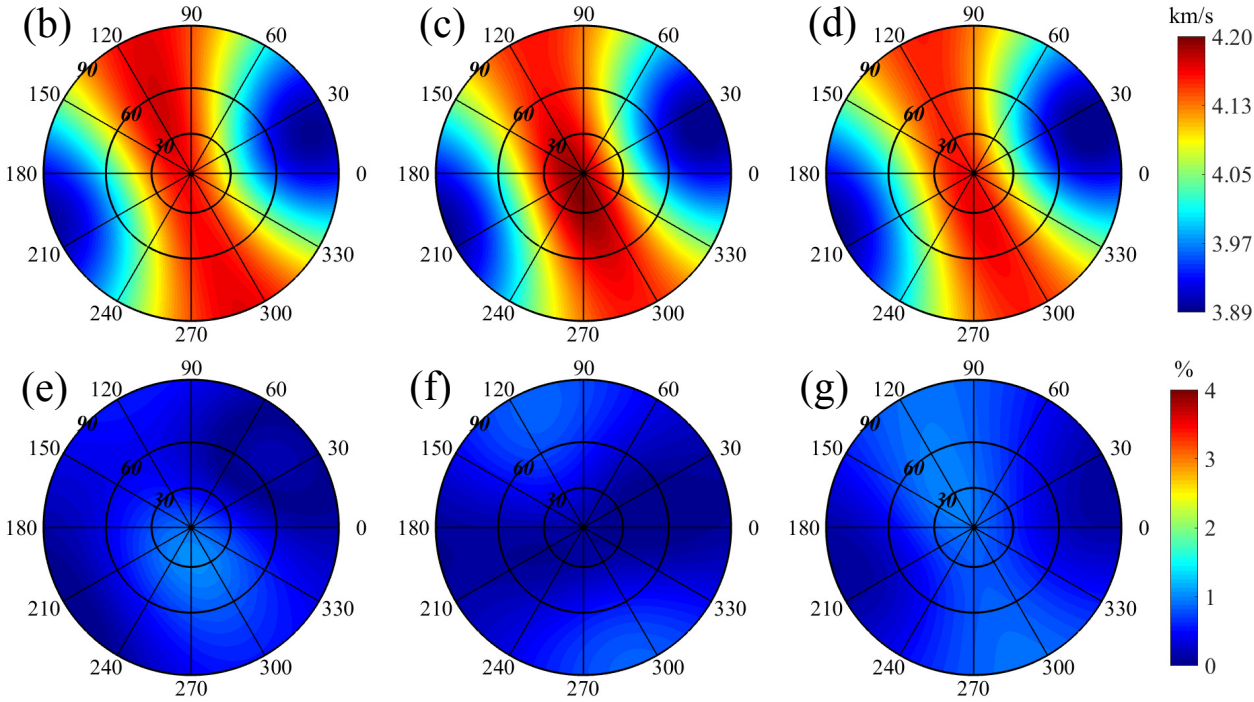

Figure 4 - Stereographic maps to the receiver in the horizontal borehole. (a) Phase velocity computed using exact WA parameters. (b) Phase velocity computed using estimated WA parameter for experiment with sources distributed along 5 profiles. (c) Phase velocity computed using estimated WA parameter for experiment with sources distributed randomly. (d) Phase velocity computed using estimated WA parameter for experiment with sources distributed in spiral pattern. (e) Percentage error between (a) and (b). (f) Percentage error between (a) and (c). (g) Percentage error between (a) and (d).

estimated parameters from data generated by sources along five profiles, randomly distributed and in spiral pattern (Figs. 4b-d, respectively), and the stereographic projection of percentage error between estimated maps and the exact one (Figs. 4e-g). Comparing the results, it is observed that estimated phase velocities and percentage error are similar for the three source distributions. Note that error does not exceed $1.5 \%$.

Figure 5 shows percentage variation maps of phase velocity. The results for sources distributed along five profiles, randomly and in spiral pattern are shown in Figures $5 a, 5 b$ and $5 c$, respectively. According to these figures, it is observed that the phase velocity suffers less variation around the $x$-axis. As elevation angle increases the velocity variation also increases. Thus, the best estimates are obtained within the region delimited by a $30^{\circ}$ cone around the borehole direction. Note that the variation is similar for the three experiments.
The covariance matrices calculated for experiments with the three sources geometries are shown in Figure 6. We note that general patterns in the three matrices are similar. Furthermore, it is observed that there is higher uncertainty in the determination of parameters $\varepsilon_{y}, \varepsilon_{z}, \delta_{x}, \delta_{y}, \delta_{z}$ and moderate to $\chi_{y}, \varepsilon_{24}$, $\varepsilon_{34}$. For the remaining parameters $\varepsilon_{x}, \chi_{y}, \chi_{z}, \varepsilon_{15}, \varepsilon_{16}, \varepsilon_{26}$ and $\varepsilon_{35}$ the variances are approximately zero. Therefore these seven parameters are accurately estimated for horizontal borehole configuration.

These results were compared with the results for a vertical borehole. Using $D\left(\alpha^{2}-\beta^{2}\right)^{-1} B_{13}-\frac{1}{2} \alpha^{-1} B_{33} \eta=D g_{i} e_{i}^{(1)}+$ $\alpha \Delta \eta$, Eq.(1) of Barreto et al. (2013). For receiver at $0.5 \mathrm{~km}$ of the vertical borehole, the Figure 7 shows the stereographic maps of phase velocity computed using exact WA parameters (Fig. 7a), using estimated parameters from data generated by sources along five profiles, randomly distributed and in spiral 
(a)
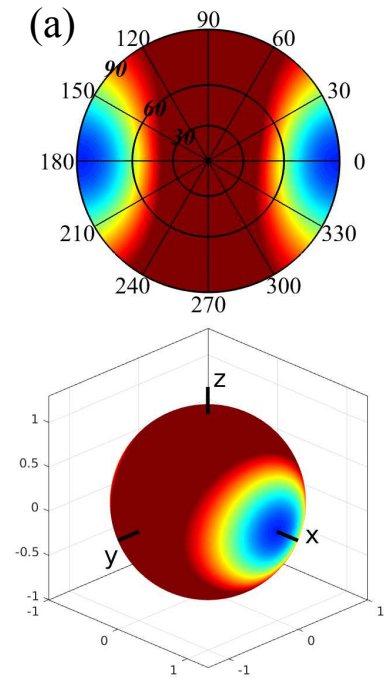

(b)
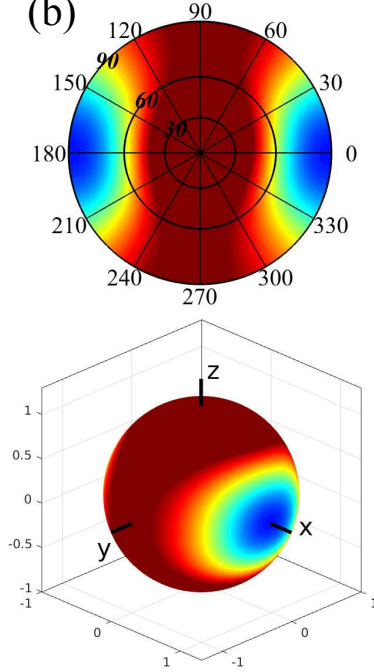

(c)
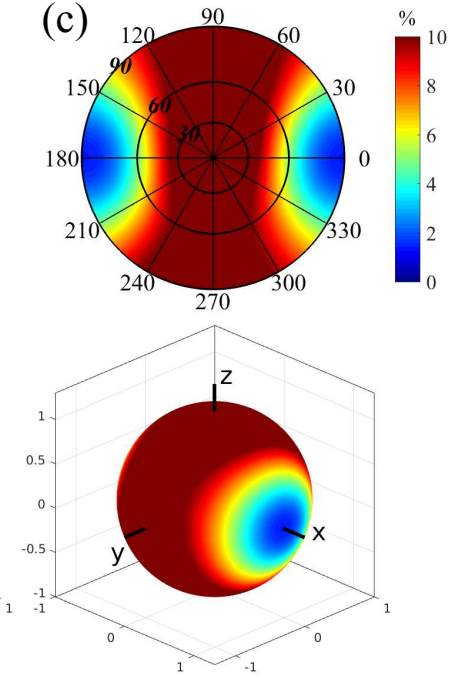

Figure 5 - Percentage variation maps (stereographic projections and its corresponding spherical surfaces) of phase velocity to the receiver in the horizontal borehole. (a) Results for sources along 5 profiles. (b) Results for sources distributed randomly. (c) Results for sources distributed in spiral pattern.

(a)

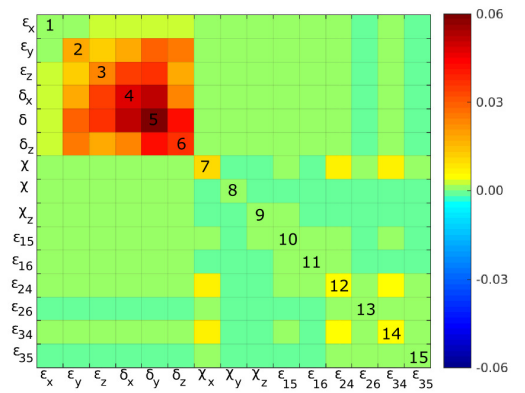

(b)

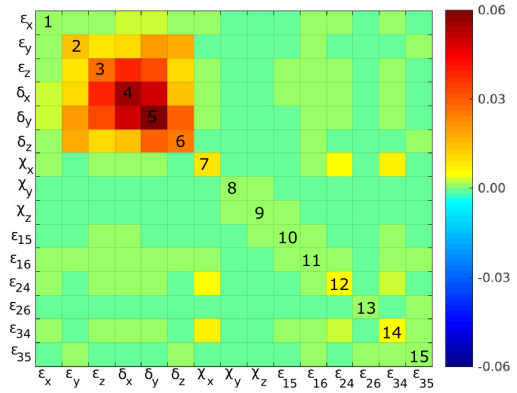

(c)

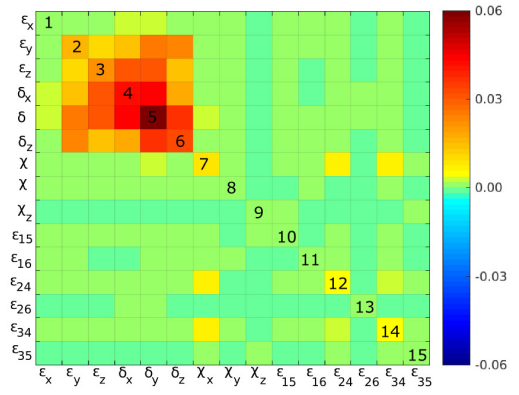

Figure 6 - Model covariance matrices computed for experiments using the three acquisition geometries to the receiver in the horizontal borehole. (a) For sources along 5 profiles. (b) For random sources. (c) For spiral sources pattern.

pattern (Figs. 7b-d, respectively) and the stereographic projection of percentage error (Figs. 7e-g) between estimated maps and the exact one. It can be seen that estimated phase velocities and percentage error are similar for the three source distributions. Note that error does not exceed $2.5 \%$.

Figure 8 shows percentage variation maps of phase velocity. The results for sources distributed along five profiles, randomly and in spiral pattern are shown in Figures 8a-c, respectively. The phase velocity suffers less variation around the vertical axis and best estimates are found within the region delimited by a $30^{\circ}$ cone. Comparing the results, it is observed that the variation is similar for the three experiments.

Figure 9 shows the computed covariance matrices for the three acquisition geometries. For the three experiments, we observe that there is greater uncertainty in the determination of parameters $\varepsilon_{x}, \varepsilon_{y}, \delta_{x}, \delta_{y}, \delta_{z}, \chi_{z}, \varepsilon_{16}$, while for the remaining parameters $\varepsilon_{z}, \chi_{x}, \chi_{y}, \varepsilon_{15}, \varepsilon_{24}, \varepsilon_{34}$ and $\varepsilon_{35}$ the variances are approximately zero. Therefore these seven parameters are accurately estimated for vertical borehole configuration.

\section{DISCUSSION}

The limited illumination of the medium together with the presence of noise in the data reduce the number of resolvable WA parameters.

For the inversion considering horizontal borehole, according to the Eq. (17), the data that effectively contribute in the inversion scheme, $\alpha \Delta \xi$, are the projection of slowness vector towards the borehole, in $x$ direction. The well 
(b)

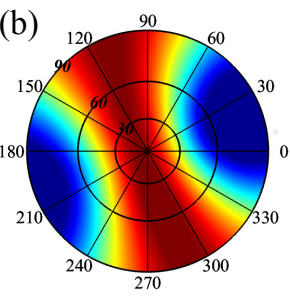

(e)

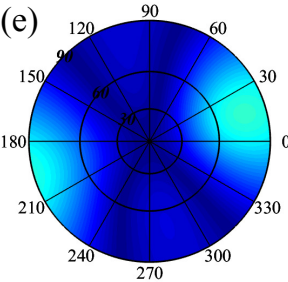

(a)

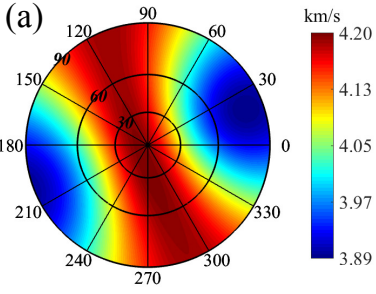

(c)

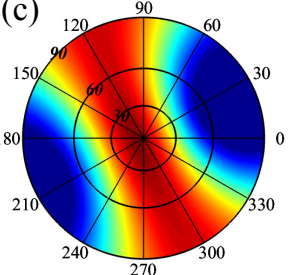

(f)

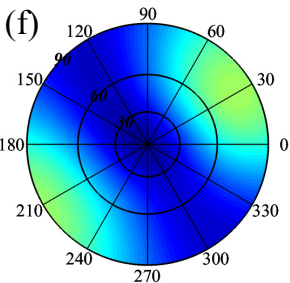

(d)

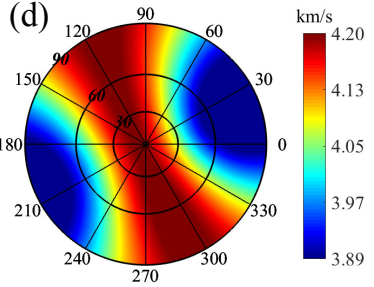

(g)

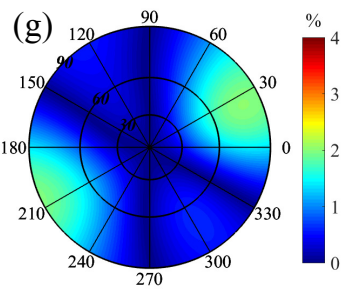

Figure 7 - Stereographic maps to the receiver of the vertical borehole. (a) Phase velocity computed using exact WA parameters. (b) Phase velocity computed using estimated WA parameter for experiment with sources distributed along 5 profiles. (c) Phase velocity computed using estimated WA parameter for experiment with sources distributed randomly. (d) Phase velocity computed using estimated WA parameter for experiment with sources distributed in spiral pattern. (e) Percentage error between (a) and (b). (f) Percentage error between (a) and (c). (g) Percentage error between (a) and (d). The $P$-wave velocities of the isotropic reference obtained for the three geometries are very close, around $4.22 \mathrm{~km} / \mathrm{s}$ for $P$-wave and $2.44 \mathrm{~km} / \mathrm{s}$ for $S$-wave.

(a)
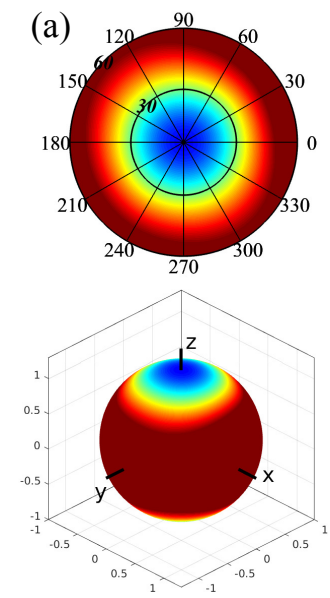

(b)
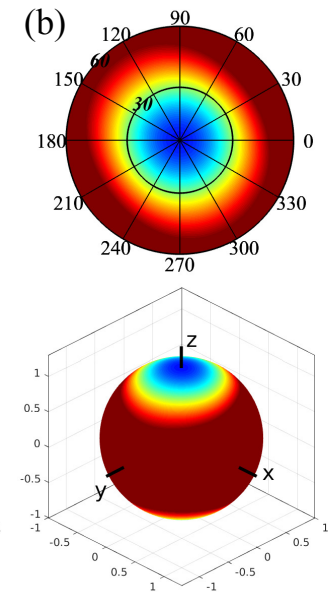

(c)
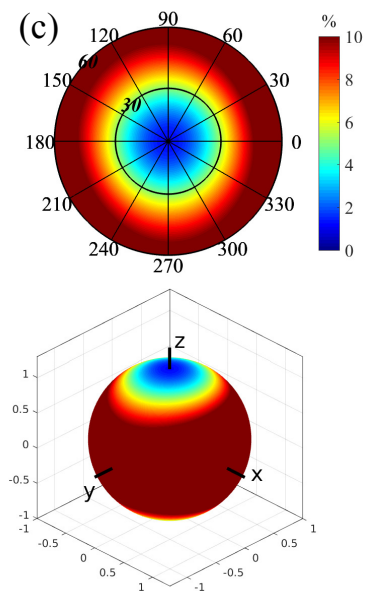

Figure 8 - Percentage variation maps (stereographic projections and its corresponding spherical surfaces) of phase velocity to the receiver of the vertical borehole. (a) Results for sources along 5 profiles. (b) Results for sources distributed randomly. (c) Results for sources distributed in spiral pattern. 
(a)

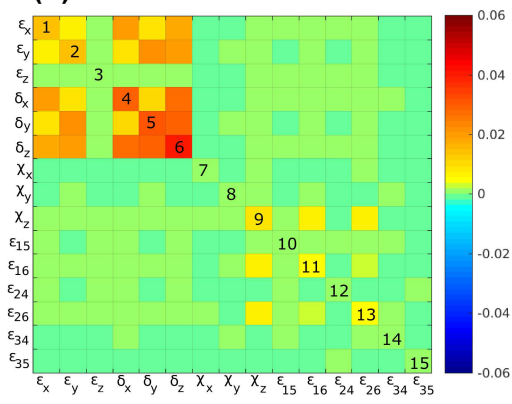

(b)

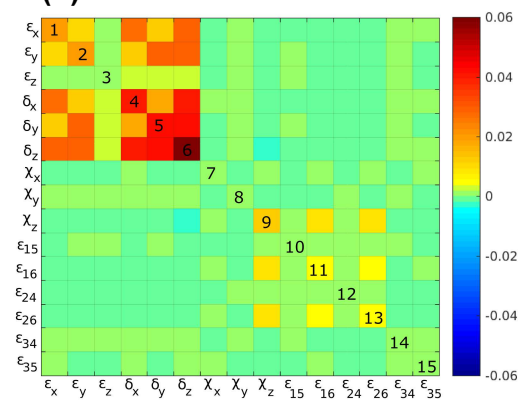

(c)

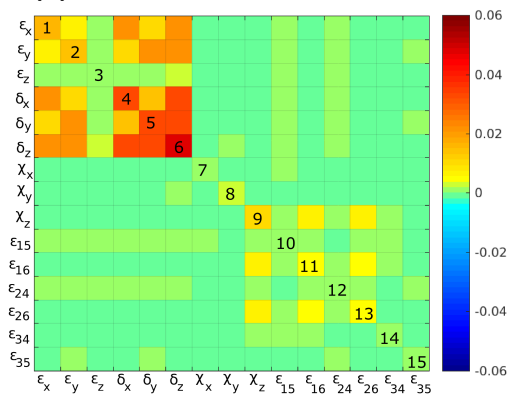

Figure 9 -Model covariance matrices computed for experiments using the three acquisition geometries to the receiver of the vertical borehole. (a) For sources along 5 profiles. (b) For random sources. (c) For spiral sources pattern.

estimated parameters for the horizontal borehole are related to the following elastic parameters of the medium, Eq. (9): $A_{11}=a_{1111}, A_{25}=a_{2213}, A_{46}=a_{2312}, A_{36}=a_{3312}, A_{45}=$ $a_{3213}, A_{15}=a_{1113}, A_{16}=a_{1112}, A_{26}=a_{2212}$ and $A_{35}=a_{3331}$. Considering an infinitesimal volume (Helbig, 1994), these indexes are related to the tension and strain tensors and the directions of application of these tensors. It is found that these parameters present at least one of the indexes in the direction ' 1 ' associated with $x$.

For the inversion considering the vertical borehole, according to Eq.(1) of Barreto et al. (2013), the data that effectively contribute to the inversion scheme, $\Delta \eta$, are to the projection of slowness vector toward the borehole, in the $z$ direction. The well estimated parameters for the vertical borehole are related to the following elastic parameters of the medium, Eq.(9): $A_{33}=a_{3333}, A_{14}=a_{1123}, A_{56}=a_{1312}, A_{25}=a_{2231}, A_{46}=$ $a_{3212}, A_{15}=a_{1113}, A_{24}=a_{2223}, A_{34}=a_{3332}$ and $A_{35}=a_{3331}$. It is found that these parameters present at least one of the indexes in the direction ' 3 ' associated with $z$.

Thus we conclude the well estimated parameters are related to borehole orientation. These conclusions are corroborated, since the parameters: $A_{25}=A_{2231}, A_{46}=A_{3212}, A_{15}=A_{1113}$ and $A_{35}=A_{3331}$, which have indexes associated with the vertical and horizontal directions are well estimated for both vertical and horizontal boreholes.

The phase velocity is well estimated for angles within about $30^{\circ}$ regardless of borehole direction, Figures 4 and 5 for horizontal borehole and Figures 7 and 8 for vertical borehole. The percentage variation of this portion of phase velocity are around $5 \%$, which we considered acceptable for this problem.

In contrast to study of Ruzek \& Pšencík (2016), here no advantages were observed in the use of randomly distributed sources on the surface.

\section{CONCLUSION}

Most of fractured hydrocarbon reservoir has a background anisotropy either by layering or by fractures with a given directional alignment. In case of horizontal borehole in a medium with fractures aligned vertically, a induced multistage fracturing can be introduced by stress shadows near to the wellbore. For this situation an enhancement on anisotropy can be observed (Murtaza et al., 2013). Our method can support a degree of weak anisotropy, i.e., our method can be efficient for total anisotropy (background + induced) until 10\% (in Thomsen's parameters).

In this work, the inversion scheme using VSP data from direct $q P$-wave for the estimation of the parameters of weak anisotropy WA is analyzed considering the orientation of the horizontal borehole.

The inversion procedure is based on a linearized model that relates weak anisotropy (WA) parameters of the medium around a borehole receiver to measurements of polarization and slowness of $q P$-wave recorded in the receiver.

We investigated the use of different types of source distribution on the surface for horizontal borehole configuration. Numerical experiments showed that, in general, the use of any the three source distributions investigated will yield similar results. Numerical tests were performed for TI medium and the synthetic data recorded in horizontal borehole. For comparison results in vertical borehole were showed, the results show that the estimation of the anisotropic parameters depends on the orientation of the borehole, however, regardless of the orientation of the borehole, the phase velocity is always well estimated in a cone of $30^{\circ}$ around the borehole.

The analysis showed that seven WA parameters can accurately estimated for both borehole configuration. By means of the estimation of these parameters one can determine the orientation of the axis of symmetry of the medium $\mathrm{TI}$ and thus 
the direction of fracture. The fracture direction is known to be the preferred direction of flow.

Beyond that, we believe that the methodology present in this work can be very useful for characterization of unconventional hydrocarbon reservoir for arbitrary anisotropic symmetry. For further work, we propose apply this methodology in a real anisotropic data set.

\section{ACKNOWLEDGMENTS}

The authors thank the Professor Ivan Pšencík and Jadsom Figueiredo for very important discussions, the Postgraduate Program in Geophysics of Universidade Federal do Pará and CAPES (a Foundation of Education Ministry of Brazil) for financial support.

\section{REFERENCES}

ASGHARZADEH M, BÓNA A, PEVZNER R, UROSEVIC M \& GUREVICH B. 2013. Reliability of the slowness and slowness-polarization methods for anisotropy estimation in VTI media from 3C walkaway VSP data. Geophysics., 78(5): WC93-WC102. doi: 10.1190/geo2012-0409.1.

ASTER RC, BORCHERS B \& THURBER CH. 2019. Parameter Estimation and Inverse Problems. Volume 90. Elsevier. 3rd ed., 404 pp.

BARRETO A, GOMES EN, MACAMBIRA RN \& COSTA JC. 2013. Improvement of local anisotropy estimation from VSP data through experimental design. Journal of Geophysics and Engineering, 10(4): 1-12. doi: 10.1088/1742-2132/10/4/045008.

BLACKBURN J, DANIELS J, DINGWALL S, HAMPDEN-SMITH G, LEANEY S, LE CALVEZ J, NUTT L, MENKITI H, SANCHEZ A \& SCHINELLI M. 2007. Borehole seismic surveys: Beyond the vertical profile. Oilfield Review, 19: 20-35.

DEWANGAN P \& GRECHKA V. 2003. Inversion of multicomponent, multiazimuth, walkaway VSP data for the stiffness tensor. Geophysics, 68(3): 1022-1031.

EHLIG-ECONOMIDES C, EBBS D \& MEEHAN DN. 1990. Factoring Anisotropy into Well Design. Oilfield Review, 2(4): 24-33.

FARRA V \& PŠENCíK I. 2003. Properties of the zeroth-, first-, and higher-order approximations of attributes of elastic waves in weakly anisotropic media. The Journal of the Acoustical Society of America, 114(3): 1366-1378.

GAISER J. 1990. Transversely isotropic phase velocity analysis from slowness estimates. Journal of Geophysical Research: Solid Earth, 95(B7): 11241-11254.
GAJEWSKI D \& PŠENCIK I. 1990. Vertical seismic profile synthetics by dynamic ray tracing in laterally varying layered anisotropic structures. Journal of Geophysical Research: Solid Earth, 95(B7): 11301-11315.

GOMES E, ZHENG X, PŠENCÍK I, HORNE S \& LEANEY S. 2004. Local Determination of Weak Anisotropy Parameters from Walkaway VSP qP-wave data in the Java Sea Region. Studia Geophysica et Geodaetica, 48(1): 215-231. doi: 10.1023/B:SGEG.0000015593.29477.88.

GRECHKA V, JORGENSEN P \& LOPEZ JL. 2006. Anisotropy Estimation from Marine 3D VSP data. In: Offshore Technology Conference, Houston, Texas, U.S.A., OTC 17866, p. 1-4.

GRECHKA V \& MATEEVA A. 2007. Inversion of P-wave VSP data for local anisotropy: Theory and case study. Geophysics, 72(4): D69-D79. doi: 10.1190/1.2742970.

HELBIG K. 1994. Foundations of Anisotropy for Exploration Seismics. Handbook of Geophysical Exploration. Volume 22. Oxford: Pergamon. $486 \mathrm{pp}$.

HORNE S \& LEANEY S. 2000. Short note: Polarization and slowness component inversion for TI anisotropy. Geophysical Prospecting, 48(4): 779-788. doi: 10.1046/j.1365-2478.2000.00205.x.

JILLEK P, HORNBY B \& RAY A. 2003. Inversion of 3D VSP P-wave data for local anisotropy: A case study. In: SEG Technical Program Expanded Abstracts 2003. p. 1322-1325. Society of Exploration Geophysicists.

MACAMBIRA RNA, GOMES ENS \& BARRETO ACR. 2014. Analysis of a linear scheme for estimation of local anisotropy from P-wave data in multi-azimuth VSP surveys. Brazilian Journal of Geophysics, 32(4): $707-720$.

MENKE W. 2012. Geophysical data analysis: discrete inverse theory. MATLAB edition. Volume 45. Academic Press. $330 \mathrm{pp}$.

MILLER DE \& SPENCER C. 1994. An exact inversion for anisotropic moduli from phase slowness data. Journal of Geophysical Research: Solid Earth, 99(B11): 21651-21657.

MURTAZA M, AL NAEIM S \& WALEED A. 2013. Design and Evaluation of Hydraulic Fracturing in Tight Gas Reservoirs. OnePetro, p. 1-10. doi: 10.2118/168100-MS.

PARSCAU J. 1991. P- and SV-wave transversely isotropic phase velocities analysis from VSP data. Geophysical Journal International, 107(3): 629-638.

PŠENCÍK I \& GAJEWSKI D. 1998. Polarization, phase velocity, and NMO velocity of qP-waves in arbitrary weakly anisotropic media. Geophysics, 63(5): 1754-1766. 
RUSMANUGROHO H \& MCMECHAN G. 2012. Sensitivity of estimated elastic moduli to completeness of wave type, measurement type, and illumination apertures at a receiver in multicomponent VSP data. Geophysics, 77(1): R1-R18. doi: 10.1190/ge02010-0410.1.

RUZEK B \& PŠENCÍK I. 2016. P-wave VSP traveltime inversion in weakly and moderately anisotropic media. Seismic Waves in Complex 3-D Structures, 26: 17-59. Available on: <http://sw3d.mff.cuni.cz/papers/ r26ip1.htm>. Access on: March 27, 2018.
THOMSEN L. 1986. Weak elastic anisotropy. Geophysics, 51(10): 1954-1966.

TSVANKIN I \& GRECHKA V. 2011. Seismology of azimuthally anisotropic media and seismic fracture characterization. Society of Exploration Geophysicists. 511 pp.

ZHENG X \& PŠENCÍK I. 2002. Local Determination of Weak Anisotropy Parameters from qP-wave Slowness and Particle Motion Measurements. Pure and Applied Geophysics, 159(7-8): 1881-1905.

Recebido em 6 janeiro, 2019 / Aceito em 24 fevereiro, 2019

Received on January 6, 2019 / accepted on February 24, 2019 